\title{
Trefoil Factor 2 Promotes Type 2 Immunity and Lung Repair through Intrinsic Roles in Hematopoietic and Nonhematopoietic Cells
}

\author{
Li-Yin Hung, ${ }^{*}$ Taylor K. Oniskey, ${ }^{*}$ Debasish Sen, ${ }^{\dagger}$ Matthew F. Krummel, ${ }^{\dagger}$ Andrew E. Vaughan, ${ }^{\ddagger}$ Noam A. Cohen, ${ }^{\S}$ and \\ De'Broski R. Herbert* \\ From the Division of Experimental Medicine* and the Department of Pathology, ${ }^{\dagger}$ University of California San Francisco, San Francisco, California; the \\ Department of Biological Sciences, ${ }^{\ddagger}$ University of Pennsylvania School of Veterinary Medicine, Philadelphia, Pennsylvania; and the Department of \\ Otorhinololaryngology, ${ }^{\S}$ University of Pennsylvania Perelman School of Medicine, Philadelphia, Pennsylvania
}

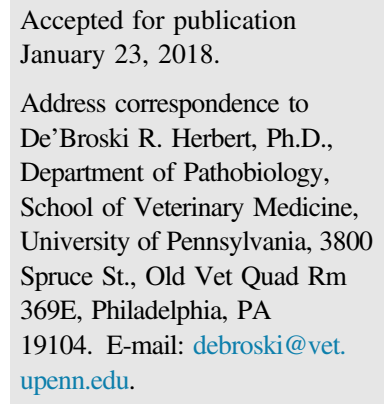

\begin{abstract}
Trefoil factors (TFFs) are small secreted proteins that regulate tissue integrity and repair at mucosal surfaces, particularly in the gastrointestinal tract. However, their relative contribution(s) to controlling baseline lung function or the extent of infection-induced lung injury are unknown issues. With the use of irradiation bone marrow chimeras, we found that TFF2 produced from both hematopoietic- and nonhematopoietic-derived cells is essential for host protection, proliferation of alveolar type 2 cells, and restoration of pulmonary gas exchange after infection with the hookworm parasite Nippostrongylus brasiliensis. In the absence of TFF2, lung epithelia were unable to proliferate and expressed reduced lung mRNA transcript levels for type 2 response-inducing IL-25 and IL-33 after infectious injury. Strikingly, even in the absence of infection or irradiation, TFF2 deficiency compromised lung structure and function, as characterized by distended alveoli and reduced blood oxygen levels relative to wildtype control mice. Taken together, we show a previously unappreciated role for TFF2, produced by either hematopoietic or nonhematopoietic sources, as a pro-proliferative factor for lung epithelial cells under steady-state and infectious injury conditions. (Am J Pathol 2018, 188: 1161-1170; https:// doi.org/10.1016/j.ajpath.2018.01.020)
\end{abstract}

Coordination of host immunity and tissue repair after infection involves a diverse array of secreted and cell-associated molecules. Indeed, mucosal cytokine production at sites of injury caused by pathogens, chemicals, and/or abrasions has a major impact on the healing process. ${ }^{1}$ TFF family proteins (TFF1 to TFF3) are reparative proteins secreted at sites of injury that drive epithelial migration over areas of denuded basement membrane, ${ }^{2}$ promote cytoskeletal rearrangement, and inhibit apoptosis. ${ }^{3,4}$ Even though trefoils are well-documented regulators of gastrointestinal injury, ${ }^{5-7}$ their role(s) in extraintestinal sites such as bladder, brain, intestine, oral cavity, kidney, liver, spleen, thymus, and lung are less well understood. ${ }^{8-12}$

Lung pathologic processes induced by allergic asthma, worm infection, smoking, tumorigenesis, chronic obstructive pulmonary disease, and naphthalene-induced lung injury all induce TFF2 production by epithelial cells. ${ }^{13-17}$ TFF2 and TFF3 can function in synergy with epidermal growth factor receptor signaling to promote bronchial epithelial cell migration $^{18}$ and coexpression of TFF1 and TFF2 in Clara cells after acute lung injury suggests that they may contribute to tissue regeneration. ${ }^{19}$ Of interest, a genetic screen identified TFF2 as a potential regulator of pulmonary function, ${ }^{20,21}$ but no evidence has found that it is important for gas exchange, tissue regeneration, or baseline lung architecture under steady-state or postinjury conditions.

Supported by NIH grants R00HL131817 (A.E.V.), R01DC013588 (N.A.C.), R01GM083204 (D.R.H.), R01AI095289 (D.R.H.), and U01AI125940 (D.R.H.), McCabe Pilot Fund Award 015802 (A.E.V.), and Veterans Administration Merit Review grant CX001617 (N.A.C.).

Disclosures: None declared. 
Both pulmonary and intestinal epithelia produce TFF2 in the context of type 2 inflammation caused by allergen or hookworm infection. ${ }^{15}$ However, the role of TFF2 as a driver or suppressor of allergic lung pathologic process is controversial. ${ }^{16,22}$ Moreover, although TFF2 expression in epithelial cells is well known, recent evidence shows that TFF2 is also expressed more broadly in the hematopoietic compartment, including $\mathrm{CD}^{+} \mathrm{T}$ cells and peritoneal macrophages. ${ }^{23}$ To determine whether hematopoietic- or nonhematopoietic-derived TFF2 was important for the production of type 2 cytokines, worm clearance, and/or restoration of lung function after infection-induced tissue damage, we turned to a Nippostrongylus brasiliensis infection model, which partially recapitulates the transient lung damage caused by human worm infections. ${ }^{24}$ Data show that hematopoietic-derived TFF2 is essential for hookworm-induced TFF2 production, host immunity, and the proliferation of alveolar type 2 cells after lung injury. Nonhematopoietic-derived TFF2 was also important for host immunity and lung tissue repair. Unexpectedly, even under homeostatic conditions without deliberate tissue damage, we found that loss of TFF2 significantly reduced pulmonary function compared with wild-type (WT) mice. Taken together, these data reveal an unexpected role for TFF2 as an essential part of lung tissue maintenance and restoration after infection in mice.

\section{Materials and Methods}

\section{Mice and N. brasiliensis Model}

All mice, including WT C57BL/6, C57BL/6 Ly5.1 (BoyJ), TFF2-deficient mice (previously backcrossed for eight generations to C57BL/6), and chicken actin-cyan fluorescent protein (CFP) transgenic mice have been previously described. ${ }^{25}$ Mice were bred in house in a fully equipped specific pathogen-free barrier facility, staffed, and maintained according to the guidelines specified by the pertinent state and federal regulations for vivaria at University of California at San Francisco or University of Pennsylvania. All procedures were reviewed and approved by Institutional Animal Care and Use Committee at University of California at San Francisco (protocol AN109782-01) or the University of Pennsylvania (protocol 805911). Sex-matched naive mice aged between 6 and 12 weeks were subcutaneously inoculated with 500 to 750 infective stage larvae collected from 7-day-old coprocultures maintained in the laboratory at $25^{\circ} \mathrm{C}$. Parasites collected in sterile $1 \times$ phosphate-buffered saline that contained penicillin/streptomycin were washed several times through repeated centrifugation before inoculation. Infected mice were euthanized by carbon dioxide narcosis at 9 days after inoculation unless specified otherwise. Adult worms were collected from the entire length of the small intestine opened longitudinally spanning the duodenum to proximal ileum and placed into a Baermann apparatus, and all of the adult worms collected after 2 to 3 hours were counted under a stereo-microscope at $\times 5$ magnification.

Bone marrow (BM) chimeras were generated between TFF2-deficient and CD45.1 age- and sex-matched strains randomly assigned as donors or recipients. The recipients were irradiated with $10 \mathrm{~Gy}$ administered as a split dose and were retro-orbitally inoculated with 2 to $5 \times 10^{6}$ total BM cells isolated from the femurs of the donor animals within 1 hour after the second dose of irradiation. All irradiated recipients were administered $1 \%$ enrofloxacin for 2 weeks after irradiation and were evaluated for chimerism efficiency at 6 to 8 weeks after transfer. Only animals that showed $>90 \%$ donor chimerism were used for subsequent data analysis.

\section{Pulse 0ximetry}

Pulse oximetry of mice were measured with MouseOx Plus with small CollarClip (both from Starr Life Sciences Corp., Oakmont, PA). Hair from the sensor site on the mice was removed 1 day before oximetry as the manufacturer's manual suggested. On the day of measurement, mice were either restrained or lightly anesthetized with ketamine/ xylazine, and 1 minute of error-free data were recorded and used in analysis. No significant differences were observed in the data readings between these two methods. Mice were kept on a heating pad $\left(37^{\circ} \mathrm{C}\right)$ during oximetry to avoid hypothermia. Results were presented as raw oxygen saturation $\left(\mathrm{SpO}_{2}\right)$, which was a readout from MouseOx Plus, or normalized to baseline $\mathrm{SpO}_{2}$, followed by calculation of the percentage of change from the initial reading in the same animal. For hookworm infection studies, mice were tattooed to ensure longitudinal data collection.

\section{Vital Imaging of Lung Slices}

For vital laser scanning confocal imaging, sections of lungs $400 \mu \mathrm{m}$ thick were prepared as previously described. ${ }^{26}$ Briefly, mice were euthanized with a lethal dose of $1.3 \%$ tribromoethanol (Avertin; Sigma-Aldrich, St. Louis, MO) and exsanguinated. Exposed lungs were then intratracheally inflated with $1.2 \mathrm{~mL}$ of $37^{\circ} \mathrm{C} 2.0 \%$ low melting temperature agarose (Fisher Scientific, Hampton, $\mathrm{NH}$ ), and solidified with cold phosphate-buffered saline $\left(8^{\circ} \mathrm{C}\right.$ to $\left.12^{\circ} \mathrm{C}\right)$. The left lobes were isolated, sliced into thick sections $(400 \mu \mathrm{m})$ with a Leica Vibratome VT1000S (Wetzler, Germany), mounted on plastic coverslips with the use of Vetbond (3M, Maplewood, $\mathrm{MN}$ ), and then imaged on a temperature-controlled stage (Warner Instruments, Hamden, CT) maintained at $37^{\circ} \mathrm{C}$, while in constant perfusion with oxygenated $(95 \%$ $\mathrm{O}_{2} ; 5 \% \mathrm{CO}_{2}$ ) RPMI without phenol red. Images were acquired with a Nikon A1R microscope (Tokyo, Japan) equipped with an automated Prior XY stage, according to the manufacturer's protocol and software, and the parameters described here. For excitation a 5-W 740- to 1000-nm tunable MaiTai HP (Newport, Santa Clara, CA) laser was used. A $20 \times 0.95$ N.A. water-immersion objective 
(Olympus XLUMPLFLN 20XW; Olympus, Tokyo, Japan) was used, and a spatial resolution of $1.13 \mu \mathrm{m} /$ pixel was achieved. Two micron Z-depth per plane was used for three-dimensional imaging. Images were recorded with the galvanometer-based scanning mode. For CFP excitation, the laser was tuned to $900 \mathrm{~nm}$ and a $450 / 50 \mathrm{~nm}$ emission band pass was used. Images were analyzed with Imaris version 7.4 (Bitplane, Concord, MA) and Matlab version R2013b (Math Works, Natick, MA). For evaluating alveolar crosssectional area, individual sections of $200 \times 200 \times 50$ $\mu \mathrm{m}^{3}$ from the three-dimensional imaging volume were selected, and alveolar area was calculated as the area of contiguous dark space or the absence of CFP with the use of Matlab scripts for each plane in the three-dimensional volume by using a cutoff $\geq 2000 \mu \mathrm{m}^{2}$.

\section{Quantitative Real-Time PCR}

Total RNA was harvested from lung or intestinal tissues with the use of RNeasy Mini kit (Qiagen, Hilden, Germany) according to the manufacturer's protocol. Total RNA (500 ng) was reverse-transcribed with Superscript II (Invitrogen, Carlsbad, CA) according to the manufacturer's protocol. One to four diluted cDNA samples were added to SsoAdavanced SYBR Green Supermix (Bio-Rad, Hercules, CA), and realtime PCR reactions were run on CFX96 Real-Time PCR detection system (Bio-Rad). Gene expression is normalized to Gapdh, and data are presented as means \pm SEM from the replicates. Primers used in this study included the following: Gapdh forward: 5'-AGGTCGGTGTGAACGGATTTG-3', and reverse: $5^{\prime}$-TGTAGACCATGTAGTTGAGGTCA-3'; Il25 forward: $5^{\prime}$-GCTGTTGCTGAAGAAGGTAGT- $3^{\prime}$, and reverse: 5'-TTCAAGTCCCTGTCCAAC-3'; Il33 forward: 5'TCCCAACAGAAGACCAAAG- $3^{\prime}$, and reverse: 5'-GATACTGCCAAGCAAGGAT-3'; Retnla forward: 5'-GGATGCCAACTTTGAATAGG-3', and reverse: 5'-GCACACCCAGTAGCAGTC-3'); Retnlb forward: 5'-GATACTGCCAAGCAAGGAT- $3^{\prime}$, and reverse: $5^{\prime}$-AACACAGTGTAGGCTTCATGCTGTA-3'; $S p c$ forward: 5'-AGCAAAGAGGTCCTGATGGA- $3^{\prime}$, and reverse: $5^{\prime}$-ATGAGAAGGCGTTTGAGGTG-3'; and Ifng forward: 5'-GTGGCATAGATGTGGAAGAA- $3^{\prime}$, and reverse: 5'-GCTGTTGCTGAAGAAGGTAGT- $3^{\prime}$.

\section{Flow Cytometry}

The following antibodies were purchased from BioLegend (San Diego, CA): rat anti-mouse CD31 (clone 390), rat anti-mouse CD45 (30-F11), rat anti-mouse CD49f/ $\alpha 6$ integrin (GoH3), rat anti-mouse CD104/ß34 integrin (346-11A), rat anti-mouse F4/80 (BM8), and rat anti-mouse CD326/epithelial cell adhesion molecule (EpCAM; G8.8). Hamster anti-mouse Podoplanin/T1a (eBio8.1.1), rat anti-mouse CD11b (M1/70), hamster anti-mouse CD11c (N418), rat anti-mouse I-A/I-E (M5/114.15.2), hamster anti-mouse CD103 (2E7), and rat antimouse Sca-1 (D7) were purchased from eBioscience (San
Diego, CA). Cell viability was determined by LIVE/DEAD Fixable Aqua Stain (Life Technologies, Carlsbad, CA) and Annexin V (BD Biosciences, San Jose, CA). Rat anti-mouse Siglec-F (E50-2440) was purchased from BD Biosciences. Flow cytometry was performed on LSRII machine (BD Biosciences) with BD FACSDiva software versions 8.0 and 8.0.1.

\section{Enzyme-Linked Immunosorbent Assay}

Mouse lungs were lavaged with $1 \mathrm{~mL}$ of phosphate-buffered saline during necropsy. After centrifugation at $1500 \mathrm{rpm}$ for 5 minutes the supernatants were stored at $-80^{\circ} \mathrm{C}$ before enzyme-linked immunosorbent assay. Mouse Trefoil Factor 2 ELISA (enzyme-linked immunosorbent assay) kit was purchased from United States Biological (Swampscott, MA).

\section{Statistical Analysis}

Sample sizes were not determined statistically before experiments. All mice were randomly assigned to experimental or control groups. All data shown are means and SEM from four to six mice per group unless otherwise indicated. Experiments were repeated at least twice with representative data shown. Grubb's test was performed for each data set to exclude outliers. For comparison unpaired $t$-test was used to compare two groups and one-way analysis of variance was used to compare three or more groups. Two-way analysis of variance was used to compare data from repeated measures of same animals for longitudinal analysis. Statistics were performed with GraphPad Prism version 6 (GraphPad Software, Inc., La Jolla, CA). In all cases $P<0.05$ was considered statistically significant.

\section{Results}

Hematopoietic Cell-Derived TFF2 Serves an Important Role in Host Defense after Infection with the Hookworm Parasite N. brasiliensis

Previous demonstration that TFF2 deficiency caused myeloid cytokine dysregulation, combined with evidence that TFF2 can be expressed in $\mathrm{CD} 4^{+} \mathrm{T}$ cells, ${ }^{15,23}$ prompted us to interrogate whether hematopoietically derived TFF2 plays a critical role in the immune response and/or resolution of lung pathologic processes after $N$. brasiliensis infection. To address this question, irradiation BM chimeras were generated between WT (CD45.1) and Tff2 ${ }^{-1-}$ (CD45.2) strains [WT $\rightarrow$ WT capable of producing both hematopoietically and nonhematopoietically derived TFF2, knockout $(\mathrm{KO}) \rightarrow$ WT only capable of producing nonhematopoietically derived TFF2, WT $\rightarrow \mathrm{KO}$ only capable of producing hematopoietically derived TFF2, and $\mathrm{KO} \rightarrow \mathrm{KO}$ unable to produce TFF2]. Six weeks after lethal whole-body irradiation (10 Gy) and BM reconstitution (Figure 1A), 
A

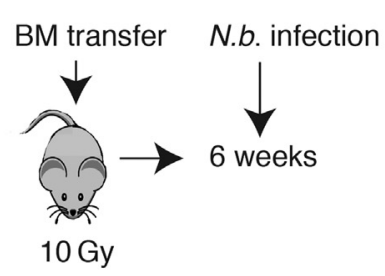

D
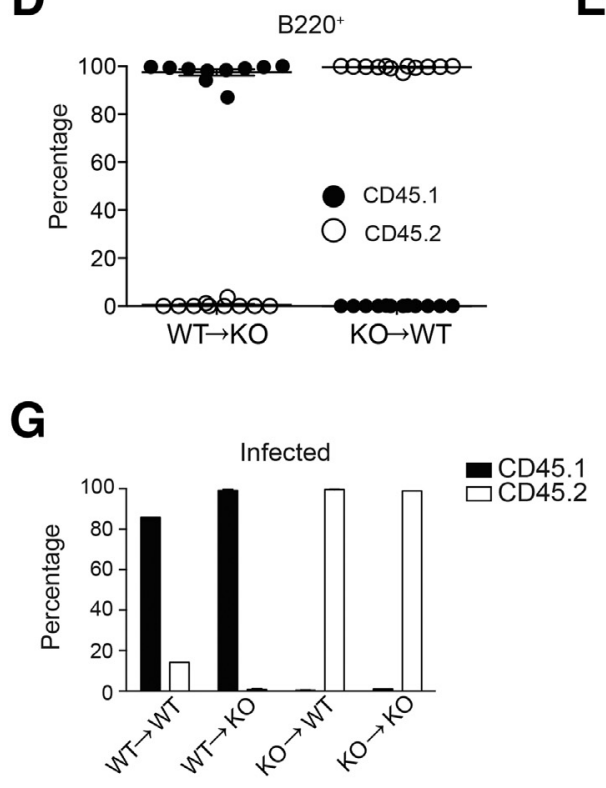

B

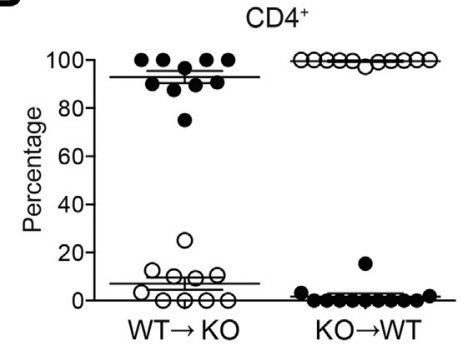

$\mathbf{E}$

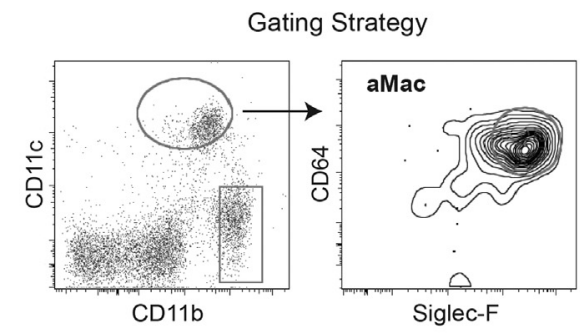

C

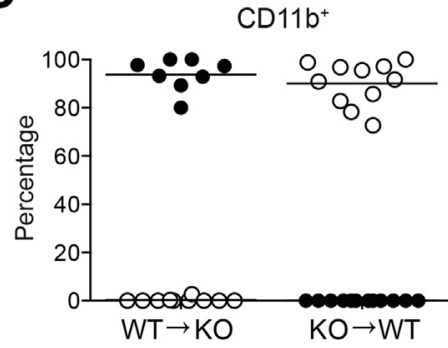

$\mathbf{F}$

Figure 1 Generation and confirmation of bone marrow (BM) chimera from wild-type (WT) and trefoil factor (TFF) $2^{-/-}$mice. A: Schematic describing irradiation and the generation of BM chimera between WT C57BL/6 (CD45.1) and Tff2 $2^{-1-}$ (CD45.2) mice. Mice were lethally irradiated, intravenously reconstituted with total BM cells from the opposite strain, rested for 6 weeks for reconstitution, followed by s.c. inoculation with 750 Nippostrongylus brasiliensis infectious larvae $L_{3}$. B-D: Chimerism in peripheral blood cells 6 weeks after initial BM transfer. Expression of congenital marker CD45.1 versus CD45.2 on $\mathrm{CD}^{+}(\mathbf{B}), \mathrm{CD}^{+} \mathrm{b}^{+}(\mathbf{C})$, and $\mathrm{B}^{2} 20^{+}$(D) cells in the blood. E: Flow gating strategy to identify alveolar macrophages (aMac) from total lung digests that are singlet, live, and $\mathrm{CD} 45^{+}$. The box indicates a gate that includes interstitial macrophages and eosinophils. $\mathbf{F}$ and $\mathbf{G}$ : Chimerism of aMac in naive $(\mathbf{F})$ and day 4 after $N$. brasiliensis infection (G). KO, knockout; N.b., N. brasiliensis.

$>95 \%$ donor chimerism was confirmed in peripheral $\mathrm{CD}^{+}$, $\mathrm{B} 220^{+}$, and $\mathrm{CD}_{11 b^{+}}$populations (Figure 1, B-D) as well as alveolar macrophages defined as $\mathrm{CD}^{2} 5^{+}, \mathrm{CD} 11 \mathrm{c}^{+}$, $\mathrm{CD}^{+} 4^{+}$, and Siglec-F ${ }^{+}$by flow cytometry with the use of monoclonal antibody specific for the congenic markers CD45.1 (WT) and CD45.2 (TFF2 ${ }^{-/-}$) (Figure 1, E-G) in all combinations tested. Therefore, TFF2 deficiency did not have a negative impact on $\mathrm{BM}$ engraftment.

To test for the relative importance of hematopoietically versus nonhematopoietically derived TFF2 in gastrointestinal host defense, the chimeric mice were inoculated with the infectious stage larvae of the hookworm parasite $N$. brasiliensis. WT $\rightarrow$ WT chimeras were significantly better protected than any other chimeric combinations at day 9 after infection, as evidenced by reduced worm burden (Figure 2A). Congruent with a host protective response, it was found that resistin-like molecule (RELM) $\beta$ intestinal mRNA transcripts were twofold to threefold higher in the $\mathrm{WT} \rightarrow$ WT chimeras than in chimeric mice lacking hematopoietically derived TFF2 $(\mathrm{KO} \rightarrow \mathrm{WT})$, nonhematopoietically derived TFF2 $(\mathrm{WT} \rightarrow \mathrm{KO})$, or both $(\mathrm{KO} \rightarrow \mathrm{KO})$ (Figure 2B).

TFF2 deficiency impaired IL33 mRNA induction in myeloid antigen-presenting cells, suggesting that TFF2 may be an important modulator of type 2 responses. ${ }^{15}$ To further investigate whether type 2 cytokine expression was impaired by TFF2 deficiency in hematopoietic or nonhematopoietic cells, lung mRNA transcripts were evaluated at day 9 by quantitative real-time RT-PCR. Transcripts for Il33 and Il25 were uniformly reduced in TFF2-deficient chimeras (Figure 2, C and D), whereas Retnla transcripts were twofold lower only in $\mathrm{KO} \rightarrow \mathrm{KO}$ and $\mathrm{KO} \rightarrow$ WT chimeras compared with $\mathrm{WT} \rightarrow \mathrm{WT}$ chimeras (Figure 2E). Conversely, lung transcripts for Ifng, the signature gene of type 1 responses, were most highly expressed in $\mathrm{KO} \rightarrow \mathrm{KO}$ chimeras, followed by $\mathrm{KO} \rightarrow \mathrm{WT}$ chimeras, and the lowest levels were observed in the $\mathrm{WT} \rightarrow \mathrm{KO}$ and $\mathrm{WT} \rightarrow \mathrm{WT}$ groups (Figure $2 \mathrm{~F}$ ). Thus, these data suggested that both hematopoietically and nonhematopoietically derived TFF2 regulate worm expulsion and substantially affect the balance between type 1 and type 2 responses during hookworm infection.

TFF2 from Either Hematopoietic or Nonhematopoietic Sources Protects against Hookworm-Induced Lung Pathologic Processes

Next, it was tested whether TFF2 was required for repair of damaged lung tissue, and, if so, whether hematopoietic or 
A

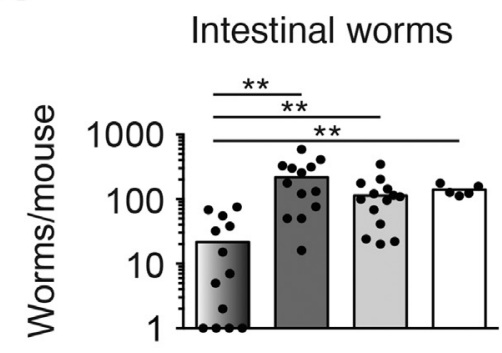

D

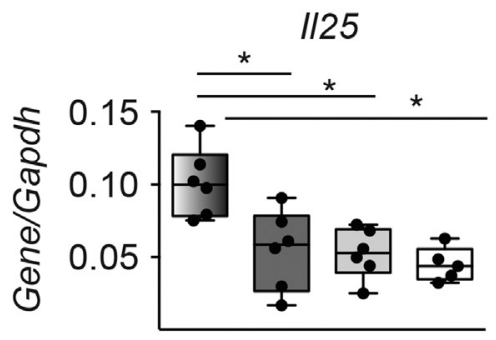

B

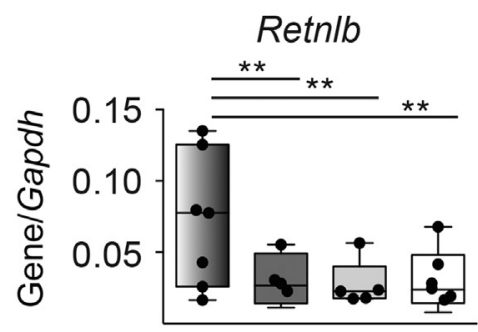

$\mathbf{E}$

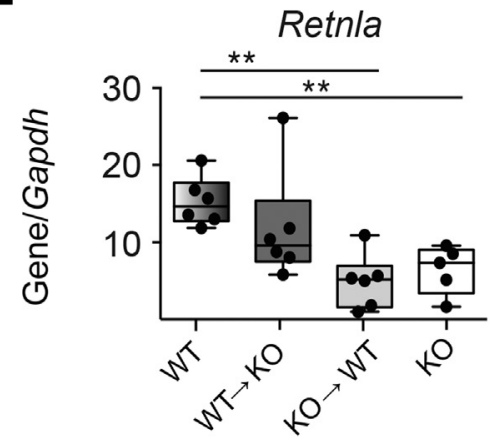

C
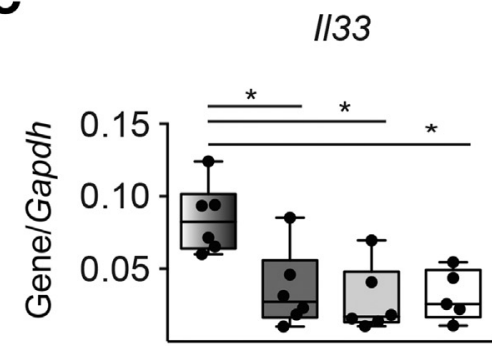

$\mathbf{F}$

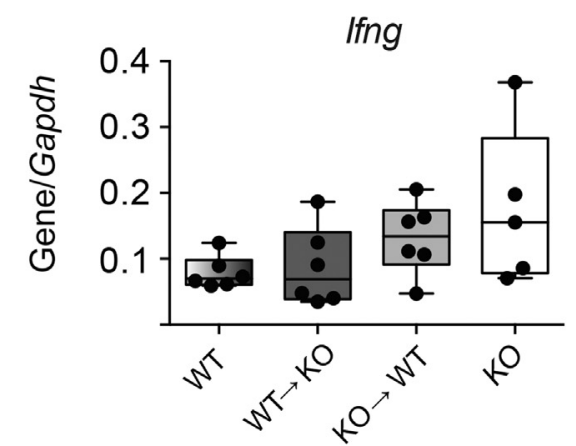

Figure 2 Defective worm expulsion, gastrointestinal resistin-like molecule $\beta$ expression, and innate type 2 cytokine production in Nippostrongylus brasiliensis-infected chimeras lacking hematopoietically or nonhematopoietically derived trefoil factor 2. A and B: Numbers of $N$. brasiliensis adult worms recovered from the intestinal lumen $(\mathbf{A})$ and Retnlb mRNA transcript levels within the jejunum of irradiation bone marrow chimeras at 9 days after infection (B). C-F: Quantitative lung mRNA expression levels of Il33 (C), Il25 (D), Retnla (E), and Ifng (F) on day 9 after N. brasiliensis infection. Data are expressed as means \pm SEM with each symbol representing one mouse. ${ }^{*} P<0.05,{ }^{*} P<0.01$ (one-way analysis of variance). K0, knockout; WT, wild-type.

nonhematopoietic sources were important. Lung damage in the infected cohorts of WT $\rightarrow \mathrm{WT}, \mathrm{KO} \rightarrow \mathrm{WT}$, WT $\rightarrow \mathrm{KO}$, and $\mathrm{KO} \rightarrow \mathrm{KO}$ chimeric mice infected as described in Mice and $\mathrm{N}$. brasiliensis Model was evaluated by pulse oximetry and histologic assessment of formalin-fixed, paraffin-embedded-inflated lungs. As shown in Figure 3A, we found elevated TFF2 levels in BALF in WT $\rightarrow$ WT cohorts on $\mathrm{d} 3$ and further increased on d9 after infection. However TFF2 levels in chimeras remained low throughout the course of infection and were lowest in $\mathrm{KO} \rightarrow \mathrm{KO}$ cohorts. Data show a similar $\mathrm{SpO}_{2}$ nadir at day 3 among strains, reflective of the peak of hookworm-induced lung injury. However, the rebound in $\mathrm{SpO}_{2}$ levels at day 9 that was observed in the WT $\rightarrow$ WT chimeras was significantly blunted in the $\mathrm{KO} \rightarrow \mathrm{WT}$ and $\mathrm{KO} \rightarrow \mathrm{KO}$ chimeras and partially impaired in the $\mathrm{WT} \rightarrow \mathrm{KO}$ chimeras (Figure 3, B and C). The histologic features of $N$. brasiliensis-infected lung tissues corroborated the persistent defects in gas exchange, with equivalent injury at day 3 (not shown), but persistent areas of denuded distal lung space lacking alveoli within both $\mathrm{KO} \rightarrow \mathrm{WT}$ and $\mathrm{KO} \rightarrow \mathrm{KO}$ chimeras (Figure 3D). Quantification of lung damage by using mean linear intercept revealed a significant reduction in $\mathrm{KO} \rightarrow \mathrm{WT}$ and $\mathrm{KO} \rightarrow \mathrm{KO}$ chimeras compared with the $\mathrm{WT} \rightarrow \mathrm{WT}$ group (Figure 3E). Intriguingly, significant differences in the number or quality of inflammatory cell infiltration in bronchoalveolar lavage fluid among chimeras and WT mice were not observed, although $\mathrm{KO}$ mice had reduced numbers of eosinophils and lymphocytes (Figure 3F). These data indicated a necessary role for both hematopoietically and nonhematopoietically derived TFF2 in the repair of lung tissue damaged by hookworm larvae.

TFF2 Is Important for Epithelial Replenishment after Lung Injury Caused by N. brasiliensis Infection

It was next determined whether the impaired $\mathrm{SpO}_{2}$ rebound and increased lung damage observed in TFF2-deficient mice were associated with defects in epithelial cell turnover. To identify S-phase lung epithelia, mice were administered bromodeoxyuridine (BrdU) 24 hours before euthanasia, and distal lung epithelia were evaluated with flow cytometry to calculate the percentage of $\mathrm{BrdU}^{+}$cells. WT $\rightarrow$ WT chimeras had higher percentages of $\mathrm{EpCAM}^{+} \mathrm{BrdU}^{+}$cells that were also pro-SpC ${ }^{+}$[surfactant protein C; alveolar type 2 (ATII) cells] compared with chimeras lacking TFF2 in either the hematopoietic or nonhematopoietic compartment (Figure 4, A and B). In addition, the total number of $\mathrm{EpCAM}^{+}$cells recovered from the distal lung compartment was higher in the WT $\rightarrow$ WT chimeras than in all other TFF2-deficient chimeras (Figure 4C). Real-time PCR analysis was used to confirm higher lung mRNA transcripts for $S p c$ (lineage marker for ATII cells) in $\mathrm{WT} \rightarrow$ WT lung compared with expression levels in WT $\rightarrow$ KO and $\mathrm{KO} \rightarrow \mathrm{KO}$ chimeras (Figure 4D). These data implied an essential role for TFF 2 for maintaining the ATII cell population in the lung after hookworm injury. 


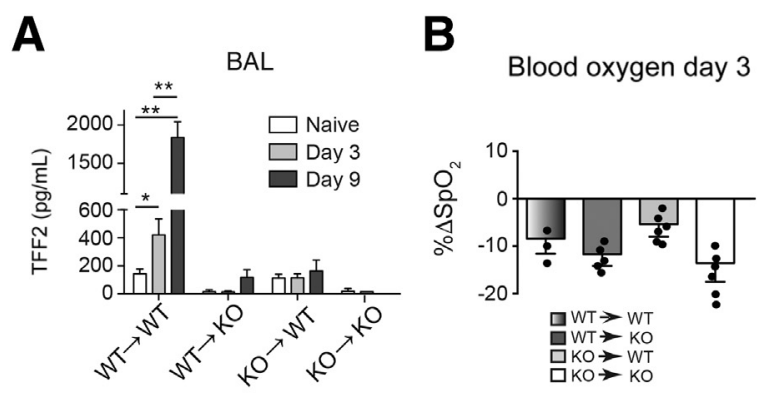

D

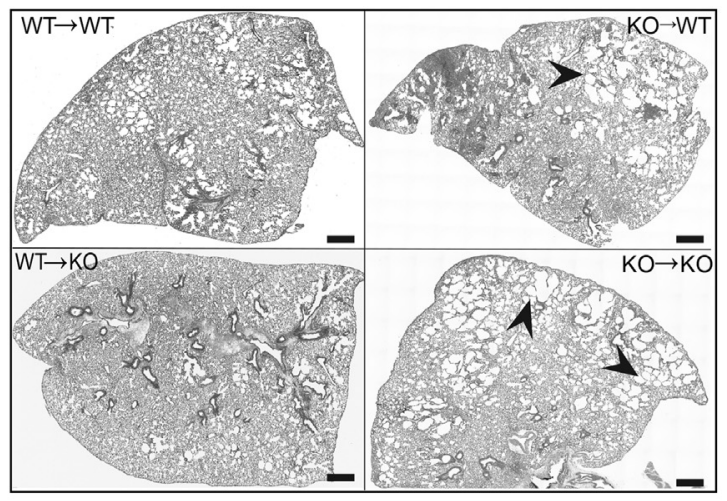

E

$\mathbf{F}$

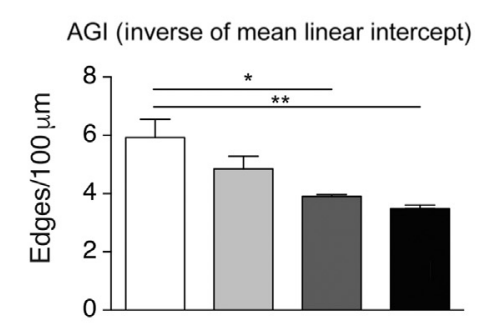

C

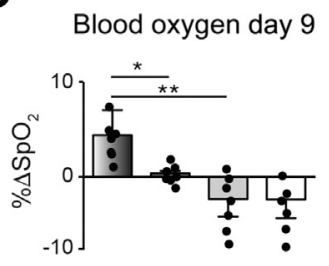

Figure 3 Both hematopoietic and nonhematopoietic sources contribute to trefoil factor (TFF)2 production, raw oxygen saturation $\left(\mathrm{SpO}_{2}\right)$ changes, and pathologic processes after Nippostrongylus brasiliensis infection. A: TFF2 levels in bronchoalveolar (BAL) fluid harvested from mice at indicated time points after $N$. brasiliensis infection. B and C: Percentage of change $\left(\Delta \mathrm{SpO}_{2}\right)$ caused by infection at 3 (B) or 9 (C) days after infection. D: Stitched images of lung tissues from chimeric mice at day 9 after $N$. brasiliensis infection. The arrowheads indicate areas of denuded distal lung space. E: Lung images (day 9) were taken and stitched as in $\mathbf{A}$ and were analyzed with AGI script. F: Differential counts of cells recovered from BAL on day 9 after $N$. brasiliensis infection. Data are expressed as means \pm SEM with each symbol representing one mouse. $n=8$ to 10 mice per group (A); $n=4$ mice per group (E); $n=4$ to 6 mice per group (F); $n=4$ independent experiments $(\mathbf{F}) .{ }^{*} P<0.05,{ }^{*} P<<0.01$ (analysis of variance). Scale bars $=0.8 \mathrm{~mm}$. Original magnification, $\times 20$. Eos, eosinophil; K0, knockout; Lymph, lymphocyte; Mac, macrophage; Neut, neutrophil; WT, wild-type.

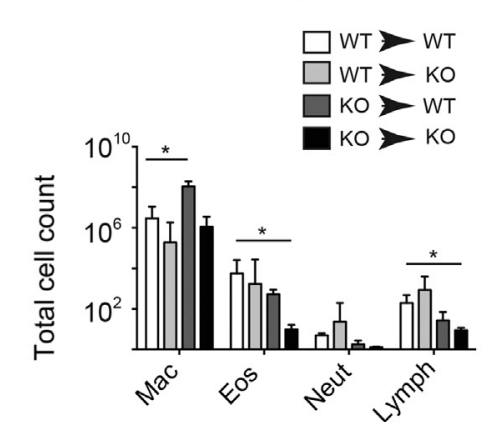

TFF2 Deficiency Leads to Reduced Gas Exchange and Abnormal Alveolar Architecture during Homeostasis

$\mathrm{KO} \rightarrow \mathrm{KO}$ chimeras consistently fared worse than all other chimeras after hookworm infection. Even under baseline conditions, the $\mathrm{SpO}_{2}$ levels in $\mathrm{KO} \rightarrow \mathrm{KO}$ chimeras were moderately lower than that of the other chimeras (Figure 5A). Given that quantitative trait loci mapping studies in mice identified TFF2 as one of several genes linked to baseline lung function, ${ }^{20,21}$ it was tested whether TFF2 deficiency, even in the absence of infectious injury, compromised lung function, and/or architecture. As expected, comparison of naive WT and $\mathrm{TFF} 2^{-1-}$ hematoxylin and eosin-stained lung tissues revealed that $\mathrm{TFF}^{-1-}$ alveoli were larger than WT alveoli (Figure 5B). To more easily visualize alveolar structure by confocal microscopy, Tff2-deficient mice were intercrossed with CFP transgenic mice ${ }^{25}$ to facilitate quantification of lung architecture by quantitative assessment of pixel intensity. Actin-CFP transgenic (WT) and TFF2 ${ }^{-1-}$ actin-CFP transgenic mice were evaluated at 6 to 10 weeks of age, and total
CFP signal was used to quantitate alveolar cross-sectional area within equal distal lung volume $(200 \times 200 \times 50$ $\mu^{3}$ ). Notably, this analysis revealed that TFF2 deficiency caused a striking increase in the frequency of large diameter alveoli compared with WT alveoli (Figure 5C). Measurement of $\mathrm{SpO}_{2}$ levels corroborated this architectural defect, as shown by significantly reduced baseline levels in TFF2deficient mice compared with WT mice under steady-state conditions (Figure 5D). Interestingly, a significant reduction was found in the number of distal lung epithelia recovered from TFF2-deficient mice compared with WT mice. Although no increase was observed in the proportion of apoptotic cells in the $\mathrm{CD}^{-} 5^{-} \mathrm{EpCAM}^{+}$population (data not shown), $\mathrm{EPCAM}^{+}$cells had lower expression of $\alpha 6 \beta 4$ integrin (Figure 6, A and B), a component of the hemidesmosome that tethers epithelia to basement membrane (Figure 6, C-F). Taken together, these data indicated that TFF2 promotes baseline lung function, promoted epithelial proliferation, and aided epithelial attachment in the pulmonary tract. 
A

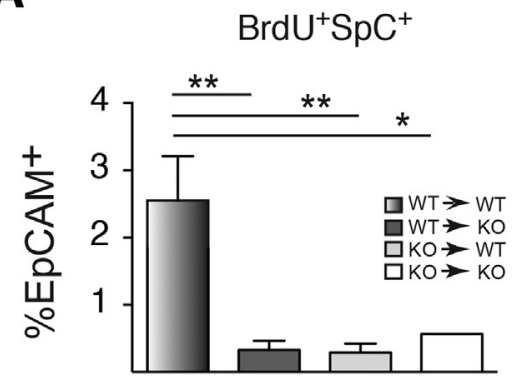

C

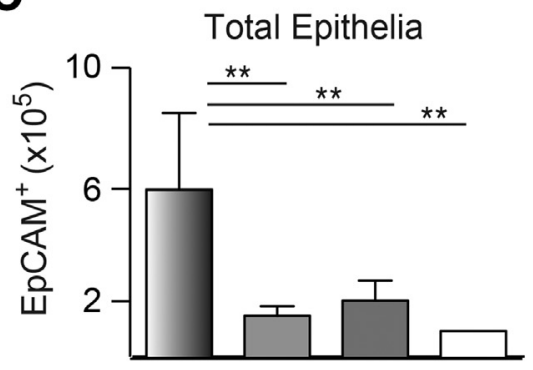

B

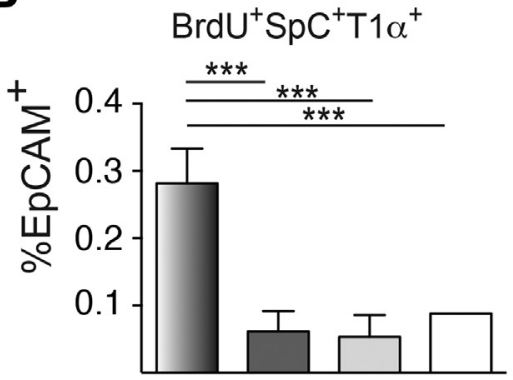

D

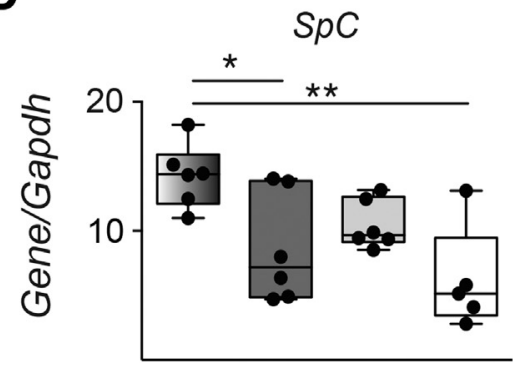

Figure 4 Decreased proliferative progenitors, total epithelial cell numbers, and $S p C$ expression in chimeric lungs after Nippostrongylus brasiliensis infection. A and B: Percentages of total numbers of the $\mathrm{CD}_{4} 5^{-}, \mathrm{EpCAM}^{+}, \mathrm{SpC}^{+}$, and $\mathrm{BrdU}^{+}(\mathbf{A})$ and $\mathrm{CD}^{-} 5^{-}, \mathrm{EpCAM}^{+}, \mathrm{SpC}^{+}, \mathrm{T} \alpha^{+}$, and $\mathrm{BrdU}^{+}(\mathrm{B})$ cell population within distal lung digests of chimeric mice at day 5 after $N$. brasiliensis infection. C: Total number of epithelia $\left(\mathrm{CD}_{4} 5^{-} \mathrm{EpCAM}^{+}\right)$cells recovered on day 5. D: Quantitative $S p C$ expression in lung tissues of chimeric mice on day 9 after $N$. brasiliensis infection. Data are expressed as means \pm SEM each symbol representing one mouse. $n=6$ to 8 mice per group $(\mathbf{A}-\mathbf{C}) ; n=4$ independent experiments $(\mathbf{A}-\mathbf{C}){ }^{*} P<0.05$, $* * P<0.01$, and $* * * P<001$ determined by analysis of variance. BrdU, bromodeoxyuridine; EpCAM, epithelial cell adhesion molecule; KO, knockout; SpC, surfactant protein C; WT, wild-type.

\section{Discussion}

TFF proteins are among the secreted factors that regulate tissue integrity and repair at mucosal surfaces, particularly in the gastrointestinal mucosa. Here, we demonstrate the importance of TFF2 as a regulator of distal lung architecture and gas exchange function. Loss of TFF2 from either hematopoietic or nonhematopoietic cells caused a reduction in type 2 cytokines (Il25, Il33), promoted Ifng expression, and abrogated epithelial cell proliferation after hookworm infection. Loss of TFF2 from all cells resulted in a marked disruption of the distal lung architecture that somewhat resembled emphysematous bullae, marked by a significant reduction in baseline blood oxygen content. Taken together, these data implicate TFF2 as an important regulator of pulmonary repair that can be derived from hematopoietic sources and that is important for maintaining mucosal homeostasis.

With the use of models of $N$. brasiliensis and house dust mite-induced type 2 responses, we previously demonstrated that TFF2-deficient mice have an early defect in the production of IL-33 and as a result show impaired
A

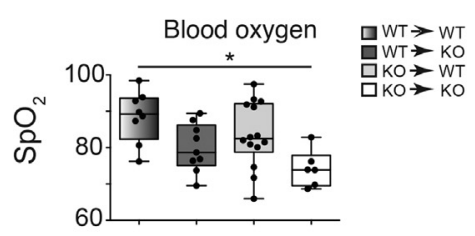

C

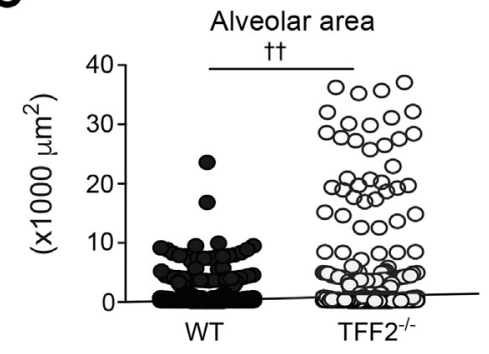

B

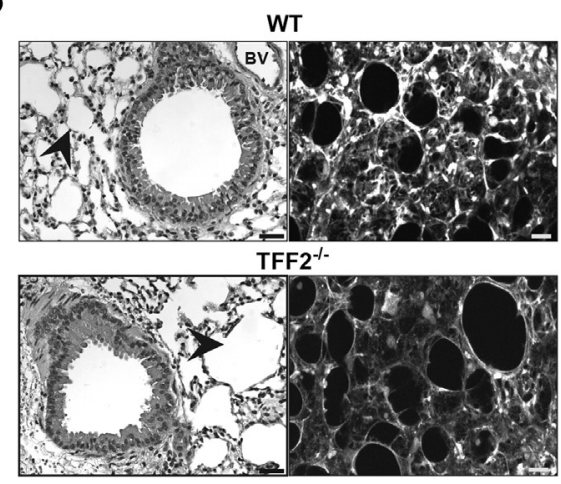

D

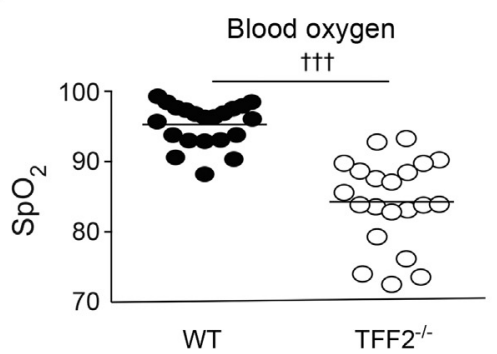

Figure 5 Trefoil factor 2 (TFF2) deficiency results in abnormally enlarged alveoli and hypoxia at the steady state. A: Measurement of baseline blood oxygen levels $\left(\mathrm{SpO}_{2}\right)$ in chimeras at 6 to 8 weeks after bone marrow reconstitution. B: Representative photomicrographs of airway and distal lung space within naive wild-type (WT) and $\mathrm{TFF}^{-/-}$mice at 6 to 8 weeks of age. Light microscopy images show paraffin-embedded hematoxylin and eosin-stained lung tissue (left) and laser-scanning confocal micrograph showing $z$ projection of gray-scale images from distal lung tissues $(50-\mu \mathrm{m}$ thick) of WT $\times$ actin-cyan fluorescent protein (CFP) transgenic mice versus Tff2 ${ }^{-1} \times$ actin-CFP transgenic naive mice (right). Arrowheads indicate alveolar space. C: Quantification of alveolar cross-sectional area in B. Each point represents area of individual alveoli. $\mathbf{D}$ : Measurement of baseline $\mathrm{Sp}_{2}$ in WT or Tff2 ${ }^{-/-}$ mice at 6 to 8 weeks of age. Each symbol represents 1 mouse (A and D). $n=3$ independent experiments (C). ${ }^{*} P<0.05$ (one-way analysis of variance); ${ }^{\dagger \dagger} P<0.01,{ }^{\dagger \dagger \dagger} P<0.001$ (unpaired $t$ test). Scale bars $=50 \mu \mathrm{m}$. Original magnification, $\times 40$. BV, blood vessel; K0, knockout. 
A

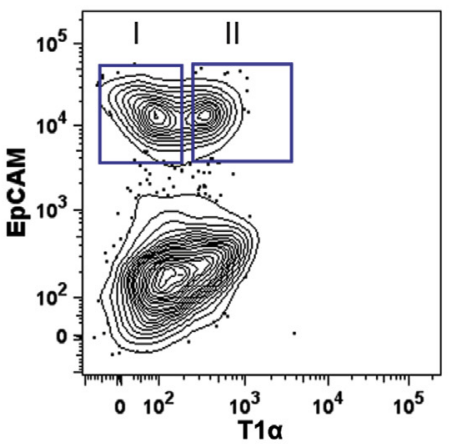

B

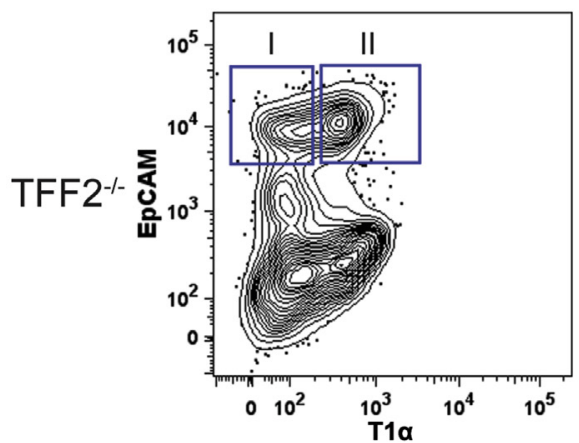

C

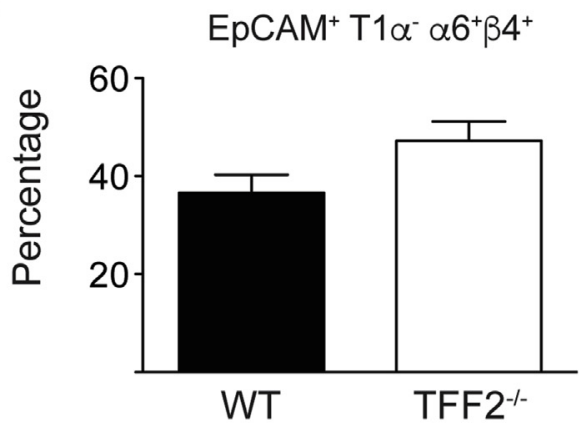

E

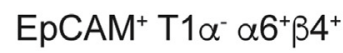

$\mathrm{EpCAM}^{+} \mathrm{T} 1 \alpha^{-} \alpha 6^{+} \beta 4^{+}$
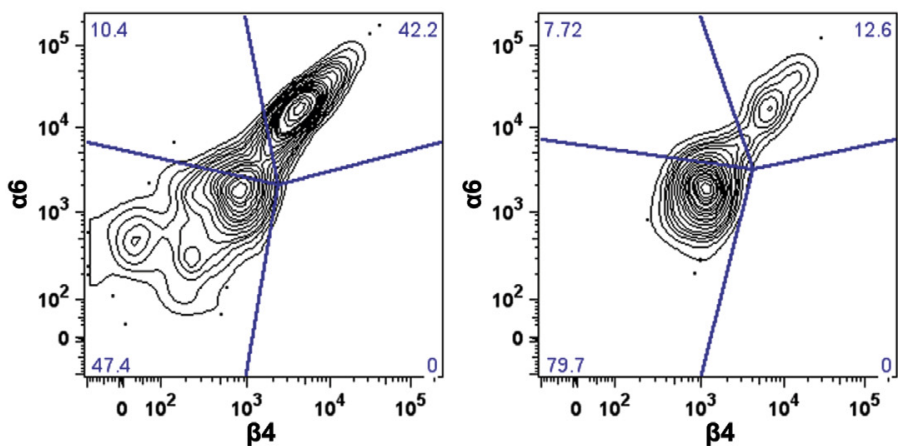

I
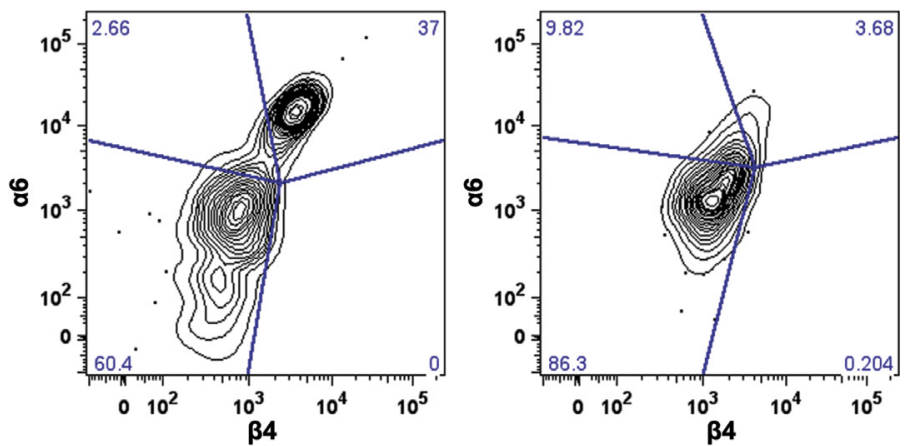

D

$\mathrm{EpCAM}^{+} \mathrm{T}^{1} \alpha^{+} \alpha 6 \beta 4^{+}$

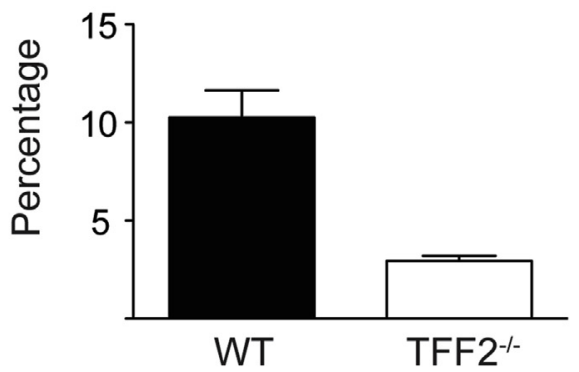

$\mathbf{F}$

$\mathrm{EpCAM}^{+} \mathrm{T} 1 \alpha^{+} \alpha 6 \beta 4^{+}$
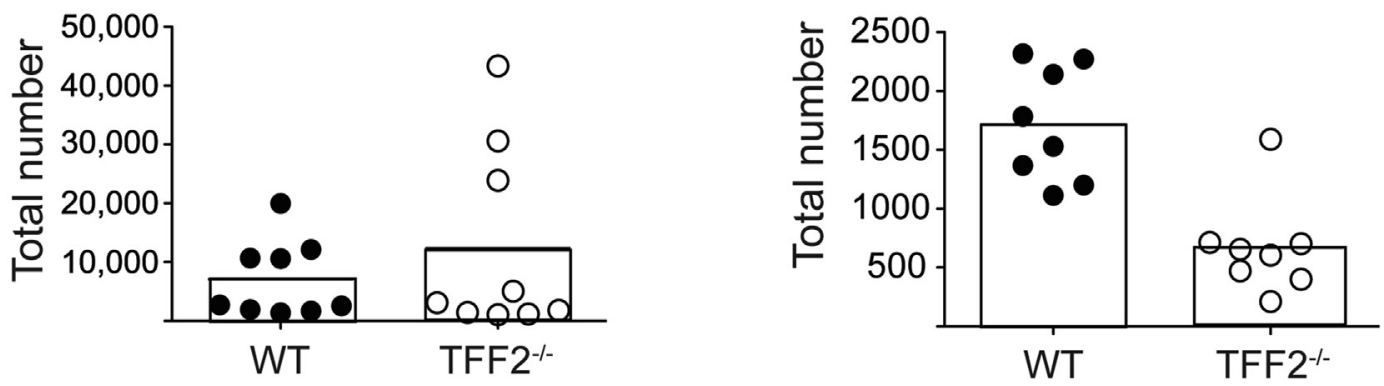

Figure 6 Trefoil factor 2 (TFF2) deficiency results in defects in epithelial cell adhesion molecules (EpCAMs) at the steady state. A and B: Representative gating strategy for the identification of alveolar type I (ATI) cells coexpressing $\alpha 6 / \beta 4$ integrins in wild-type (WT) (A) and Tff $2^{-1-}$ (B) mice. Gate I indicates $\mathrm{T}_{1} \alpha^{-} \mathrm{EpCAM}^{+}$; Gate II, $11 \alpha^{+} \mathrm{EpCAM}^{+}$. The blue lines in $\mathbf{A}$ and $\mathbf{B}$ indicate quadrants separated on the basis of the expression of integrins $\alpha 6$ and $\beta 4$ in both Gate I and Gate II. The analysis and gating were performed with FlowJo version 9.9.6 (TreeStar Inc., Ashland, OR). C-F: Percentage (C and D) and total cell numbers (E and $\mathbf{F})$ of $\mathrm{CD}_{4} 5^{-}, \mathrm{EpCAM}^{+}, \mathrm{T} 1 \alpha^{-}, \alpha 6^{+} \beta 4^{+}(\mathbf{C}$ and $\mathbf{E})$ and $\mathrm{CD} 45^{-}, \mathrm{EpCAM}^{+}, \mathrm{T} 1 \alpha^{+}, \alpha 6^{+} \beta 4^{+}(\mathbf{D}$ and $\mathbf{F})$ populations within distal lungs of naive WT and $\mathrm{Tff}^{-/-}$mice. Data are expressed as means \pm SEM with each symbol representing one mouse. $n=6$ to 8 mice per group (C and D). 
IL-13-dominated inflammatory responses. ${ }^{1}$ IL-33, a prototypical alarmin cytokine, is thought to be primarily produced by epithelial cells after tissue damage. ${ }^{2}$ In addition to epithelial cells, macrophages and inflammatory dendritic cells isolated from TFF2-deficient mice have compromised IL-33 expression. ${ }^{1}$ Myeloid-derived IL-33 has been reported by several other groups in the setting of allergic disease., Herein, we show by using irradiation BM chimeras that both Il25 and Il33 expression levels were reduced in lung myeloid cells from mice lacking BM-derived TFF2. In addition, there was a selective reduction in Retlna, which is characteristic of M2/alternatively activated macrophages, in mice lacking BM-derived TFF2. Together, this suggests that paracrine or autocrine signaling of TFF2 in hematopoietic cells favors type 2 responses. Congruent with this interpretation, expression levels for Ifng, the prototypical type 1 inflammatory cytokine, were highest in the lungs of mice lacking TFF2 in all cells and were also significantly elevated in mice lacking either BM- or non-BM-derived TFF2 compared with WT chimeras. Our data are consistent with a demonstration that $I l 33$ expression was significantly reduced in the gastrointestinal tract of TFF2-deficient animals during dextran sulfate-induced colitis ${ }^{5}$ and previous reports of TFF2-deficient animals producing enhanced proinflammatory cytokines (Ifng and Illb) ${ }^{6,7}$

Data suggest that TFF2 promotes type 2 macrophage responses and reduces type 1 responses. Although it has been shown that TFF2 deficiency leads to an enhanced M1/classically activated macrophage phenoptype, ${ }^{8}$ rTFF 2 treatment that directs macrophages to adopt an M2 phenotype in vitro could not be demonstrated (data not shown). Such results suggest that TFF2 effects on macrophages may be indirect. Because a receptor for TFF2 has not yet been identified, determining the cellular targets of TFF2 signaling remains challenging. It has been reported that CXCR4 may function as a low-affinity receptor for TFF2 in epithelia and lymphocytes, ${ }^{9}$ but it was found that myeloid-specific CXCR4-deficient mice (LysM${ }^{\mathrm{Cre}}{ }_{\mathrm{CXCR}} 4^{\text {flox/flox }}$ ) had no defects in type 2 immunity or worm expulsion during $N$. brasiliensis infection (data not shown). Further functional studies will be greatly aided by definitive identification of the TFF2 receptor.

The hematopoietic cell(s) sources of TFF2 were not formally identified in this study, but could be of myeloid lineage (macrophage) or lymphoid lineage $\left(\mathrm{CD}^{+} \mathrm{T}\right.$ cells). ${ }^{7,10}$ Tissue macrophages, which are widely accepted as central players in wound healing/tissue repair processes, express TFF2 transcripts, but the functional importance of myeloid-derived TFF2 awaits further study. Recently, Dubeyovskova et al $^{10}$ demonstrated that mature memorylike $\mathrm{CD}^{+} \mathrm{T}$ cells could produce TFF2, which is not entirely without precedent, given that $\mathrm{T}$ regulatory cells and $\mathrm{T}$ helper 2 effector cells can produce amphiregulin, an epidermal growth factor receptor agonist and pro-repair molecule. ${ }^{11-13}$ We speculate that hematopoietic cell-derived TFF2 may function similar to amphiregulin, given that TFF2-mediated epithelial migration partially requires an intact epidermal growth factor receptor. Future studies will need to discern whether TFF2 is an important product of tissue macrophages, innate lymphoid cells, and/ or Foxp $3^{+} \mathrm{T}$ regulatory cells. ${ }^{14-16}$

In the first 3 days of infection, hookworm larvae cause hemorrhagic injury to lung parenchyma because of larval migratory behavior and their secreted proteolytic enzymes. Loss of either BM- or non-BM-derived TFF2 exacerbated lung pathologic processes and impaired the proliferative expansion of lung epithelia during the repair phase. Type II alveolar epithelial cells, which coexpress EpCAM and surfactant protein $\mathrm{C}$, function as progenitors in the distal lung compartment. ${ }^{17}$ Loss of either BM- or non-BM-derived TFF2 reduced both the number and percentage of ATII cells in S phase at day 9 after infection. This time point is 6 days after worm egress from the lung and likely corresponds to a period of peak lung healing, based on the high levels of type 2 cytokine production at this time. ${ }^{18}$ Moreover, at 9 days after infection, WT mice completely recover their initial blood oxygen levels and have threefold to fourfold greater numbers of S-phase ATII distal lung epithelia than all other chimeric strains, consistent with increased $S p c$ expression levels in $\mathrm{WT} \rightarrow \mathrm{WT}$ chimeras relative to $\mathrm{KO} \rightarrow \mathrm{WT}$ or KO $\rightarrow$ KO chimeras. Scott and colleagues ${ }^{19,20}$ and Marsland et $\mathrm{al}^{21}$ demonstrated that the architectural defects caused by hookworm infection last for $>1$ year and that local lung M2 cell activation persists during this time, despite the continued emphysematous changes. However, none of these studies evaluated the percentage or number of proliferating epithelia or blood oxygen content before or after hookworm infection. ${ }^{21}$ From our findings, we surmise that TFF2 drives local epithelial proliferation to rapidly restore the lung function and blood oxygen levels despite ongoing architectural defects. The possibility that the dysregulation of inflammation in the absence of TFF2 also contributed to the reduced $\mathrm{SpO}_{2}$ levels and that this reduction was not solely due to architectural defects from reduced epithelial proliferation cannot be ruled out.

Despite the widespread notion that M2 cells promote wound healing, few studies have demonstrated that reepithelialization is macrophage dependent. ${ }^{22}$ One recent study demonstrated that co-culture of ATII cells and BMderived macrophage yielded more colony-forming units than when ATII cells are cultured alone. ${ }^{23}$ Our work provides a potential mechanism to explain how that could occur, in as much as secreted macrophages (or other hematopoietic cells) may have an important role in promoting lung repair. ${ }^{24}$ Given that alveolar macrophages are in close contact with ATII epithelial cells in vivo, we speculate that alveolar macrophages facilitate epithelial proliferation and tissue repair through a TFF2-dependent mechanism.

Interestingly, even in the absence of infectious injury or irradiation, TFF2 deficiency in mice led to a striking reduction in cell number within the distal lung compartment. This reduced cell number was linked to reduced numbers of epithelial cells expressing $\alpha 6 / \beta 4$ integrin, an essential 
component of the hemidesmosome that tethers epithelia to the basement membrane. The hemidesmosome facilitates epithelial cell attachment to basement membrane through binding laminin, which, in turn, facilitates a cascade of intracellular events that promote survival, growth, and differentiation. $^{25}$ Indeed, this signaling cascade may partially underlie the association of TFF2 with tumor cell metastasis. ${ }^{26}$

\section{Conclusions}

This work demonstrates a previously unappreciated and critical role for hematopoietic TFF2 in the development of type 2 immune responses, host protection, and ATII proliferation. In addition, demonstration that TFF2 deficiency leads to lung function defects in the absence of deliberate injury implies a broader role for TFF2 than previously thought. Together, these findings further emphasize the importance of identifying and validating molecular pathways responsible for the downstream effects of this important mucosal cytokine.

\section{Acknowledgments}

L.-Y.H. and D.R.H. designed the experiments and wrote the manuscript; L.-Y.H., T.K.O., and D.S. performed experiments and analyzed data; A.E.V. and N.A.C. cowrote the manuscript; M.F.K. provided actin-cyan fluorescent protein mice and assisted with confocal imaging.

\section{References}

1. Wills-Karp M, Rani R, Dienger K, Lewkowich I, Fox JG, Perkins C, Lewis L, Finkelman FD, Smith DE, Bryce PJ, Kurt-Jones EA, Wang TC, Sivaprasad U, Hershey GK, Herbert DR: Trefoil factor 2 rapidly induces interleukin 33 to promote type 2 immunity during allergic asthma and hookworm infection. J Exp Med 2012, 209: $607-622$

2. Van Dyken SJ, Nussbaum JC, Lee J, Molofsky AB, Liang HE, Pollack JL, Gate RE, Haliburton GE, Ye CJ, Marson A, Erle DJ, Locksley RM: A tissue checkpoint regulates type 2 immunity. Nat Immunol 2016, 17:1381-1387

3. Tjota MY, Hrusch CL, Blaine KM, Williams JW, Barrett NA, Sperling AI: Signaling through FcRgamma-associated receptors on dendritic cells drives IL-33-dependent TH2-type responses. J Allergy Clin Immunol 2014, 134:706-713.e8

4. Tjota MY, Williams JW, Lu T, Clay BS, Byrd T, Hrusch CL, Decker DC, de Araujo CA, Bryce PJ, Sperling AI: IL-33-dependent induction of allergic lung inflammation by FcgammaRIII signaling. J Clin Invest 2013, 123:2287-2297

5. Judd LM, Chalinor HV, Walduck A, Pavlic DI, Dabritz J, Dubeykovskaya Z, Wang TC, Menheniott TR, Giraud AS: TFF2 deficiency exacerbates weight loss and alters immune cell and cytokine profiles in DSS colitis, and this cannot be rescued by wild-type bone marrow. Am J Physiol Gastrointest Liver Physiol 2015, 308:G12-G24

6. McBerry C, Egan CE, Rani R, Yang Y, Wu D, Boespflug N, Boon L, Butcher B, Mirpuri J, Hogan SP, Denkers EY, Aliberti J, Herbert DR: Trefoil factor 2 negatively regulates type 1 immunity against Toxoplasma gondii. J Immunol 2012, 189:3078-3084

7. Kurt-Jones EA, Cao L, Sandor F, Rogers AB, Whary MT, Nambiar PR, Cerny A, Bowen G, Yan J, Takaishi S, Chi AL, Reed G,
Houghton J, Fox JG, Wang TC: Trefoil family factor 2 is expressed in murine gastric and immune cells and controls both gastrointestinal inflammation and systemic immune responses. Infect Immun 2007, 75: 471-480

8. Giraud AS, Pereira PM, Thim L, Parker LM, Judd LM: TFF-2 inhibits iNOS/NO in monocytes, and nitrated protein in healing colon after colitis. Peptides 2004, 25:803-809

9. Dubeykovskaya Z, Dubeykovskiy A, Solal-Cohen J, Wang TC: Secreted trefoil factor 2 activates the CXCR4 receptor in epithelial and lymphocytic cancer cell lines. J Biol Chem 2009, 284: $3650-3662$

10. Dubeykovskaya Z, Si Y, Chen X, Worthley DL, Renz BW, Urbanska AM Hayakawa Y, Xu T, Westphalen CB, Dubeykovskiy A, Chen D, Friedman RA, Asfaha S, Nagar K, Tailor Y, Muthupalani S, Fox JG, Kitajewski J, Wang TC: Neural innervation stimulates splenic TFF2 to arrest myeloid cell expansion and cancer. Nat Commun 2016, 7:10517

11. Zaiss DM, van Loosdregt J, Gorlani A, Bekker CP, Grone A, Sibilia M, van Bergen en Henegouwen PM, Roovers RC, Coffer PJ, Sijts AJ: Amphiregulin enhances regulatory $\mathrm{T}$ cell-suppressive function via the epidermal growth factor receptor. Immunity 2013, 38:275-284

12. Zaiss DM, Yang L, Shah PR, Kobie JJ, Urban JF, Mosmann TR Amphiregulin, a $\mathrm{TH} 2$ cytokine enhancing resistance to nematodes. Science 2006, 314:1746

13. Arpaia N, Green JA, Moltedo B, Arvey A, Hemmers S, Yuan S, Treuting PM, Rudensky AY: A distinct function of regulatory T cells in tissue protection. Cell 2015, 162:1078-1089

14. Monticelli LA, Osborne LC, Noti M, Tran SV, Zaiss DM, Artis D: IL 33 promotes an innate immune pathway of intestinal tissue protection dependent on amphiregulin-EGFR interactions. Proc Natl Acad Sci U S A 2015, 112:10762-10767

15. Burzyn D, Kuswanto W, Kolodin D, Shadrach JL, Cerletti M, Jang Y, Sefik E, Tan TG, Wagers AJ, Benoist C, Mathis D: A special population of regulatory T cells potentiates muscle repair. Cell 2013, 155:1282-1295

16. Meng C, Liu G, Mu H, Zhou M, Zhang S, Xu Y: Amphiregulin may be a new biomarker of classically activated macrophages. Biochem Biophys Res Commun 2015, 466:393-399

17. Barkauskas CE, Cronce MJ, Rackley CR, Bowie EJ, Keene DR, Stripp BR, Randell SH, Noble PW, Hogan BL: Type 2 alveolar cells are stem cells in adult lung. J Clin Invest 2013, 123:3025-3036

18. Chen F, Liu Z, Wu W, Rozo C, Bowdridge S, Millman A, Van Rooijen N, Urban JF Jr, Wynn TA, Gause WC: An essential role for TH2-type responses in limiting acute tissue damage during experimental helminth infection. Nat Med 2012, 18:260-266

19. Siracusa MC, Reece JJ, Urban JF Jr, Scott AL: Dynamics of lung macrophage activation in response to helminth infection. J Leukoc Biol 2008, 84:1422-1433

20. Reece JJ, Siracusa MC, Southard TL, Brayton CF, Urban JF Jr, Scott AL: Hookworm-induced persistent changes to the immunological environment of the lung. Infect Immun 2008, 76:3511-3524

21. Marsland BJ, Kurrer M, Reissmann R, Harris NL, Kopf M: Nippostrongylus brasiliensis infection leads to the development of emphysema associated with the induction of alternatively activated macrophages. Eur J Immunol 2008, 38:479-488

22. Martinez FO, Sica A, Mantovani A, Locati M: Macrophage activation and polarization. Front Biosci 2008, 13:453-461

23. Lechner AJ, Driver IH, Lee J, Conroy CM, Nagle A, Locksley RM, Rock JR: Recruited monocytes and type 2 immunity promote lung regeneration following pneumonectomy. Cell Stem Cell 2017, 21:120-134.e7

24. Herold S, Mayer K, Lohmeyer J: Acute lung injury: how macrophages orchestrate resolution of inflammation and tissue repair. Front Immunol 2011, 2:65

25. Mercurio AM, Rabinovitz I, Shaw LM: The alpha 6 beta 4 integrin and epithelial cell migration. Curr Opin Cell Biol 2001, 13:541-545

26. Dhar DK, Wang TC, Maruyama R, Udagawa J, Kubota H, Fuji T, Tachibana M, Ono T, Otani H, Nagasue N: Expression of cytoplasmic TFF2 is a marker of tumor metastasis and negative prognostic factor in gastric cancer. Lab Invest 2003, 83:1343-1352 